\title{
LÍQUENES EPIFÍTICOS DE ALGUNAS LOCALIDADES COSTERAS DE MARRUECOS
}

\author{
F. Leandro ALONSO \& José M. EGEA
}

\begin{abstract}
RESUMEN. Líquenes epifíticos de algunas localidades costeras de Marruecos. Estudio florístico de los líquenes epifíticos recolectados en varias localidades costeras de Marruecos. Se citan 61 taxones de los cuales son nuevas citas para Marruecos: Arthothelium sardoum, Bacidia igniarii, B. laurocerasi, Bacidina phacodes, Bactrospora patellarioides var. convexa, Cladonia coniocraea, Lecanora horiza, Pertusaria coccodes, Physconia enteroxantha, Rinodina anomala y $R$. oleae.
\end{abstract}

Palabras clave. Líquenes, epífitos, flora, costa, litoral, Marruecos.

ABSTRACT. Epiphytic lichens of some coastal localities of Morocco. Floristic study of the epiphytic lichens from some coastal localities in Morocco. 61 taxa are presented. New record from Morocco are: Arthothelium sardoum, Bacidia igniarii, B. laurocerasi, Bacidina phacodes, Bactrospora patellarioides var. convexa, Cladonia coniocraea, Lecanora horiza, Pertusaria coccodes, Physconia enteroxantha, Rinodina anomala and $R$. oleae.

Key words. Lichen, epiphyte, flora, coastal, litoral, Morocco.

\section{INTRODUCCIÓN}

Continuando con la línea de investigación sobre la flora de Marruecos, presentamos los resultados obtenidos en el estudio de los líquenes epífitos de diversos puntos de las costas de Marruecos (fig. 1). En un trabajo anterior sobre líquenes de rocas calizas y terrícolas de Marruecos (Alonso y Egea, 1994), se ofrecen datos sobre la situación, clima y geología de las localidades estudiadas. En Egea (1996) se presenta una aproximación al catálogo de hongos liquenizados y liquenícolas de Marruecos, y se hace referencia a estudios anteriores realizados en este país.

Este artículo debe entenderse como una aportación, ya que en ninguna de las localidades se ha hecho una recolección exahustiva de material.

Este trabajo se ha realizado en el marco del proyecto de investigación PB 93-1129-C02-01, financiado por la Dirección General de Investigación Científica y Técnica (DGICYT) 


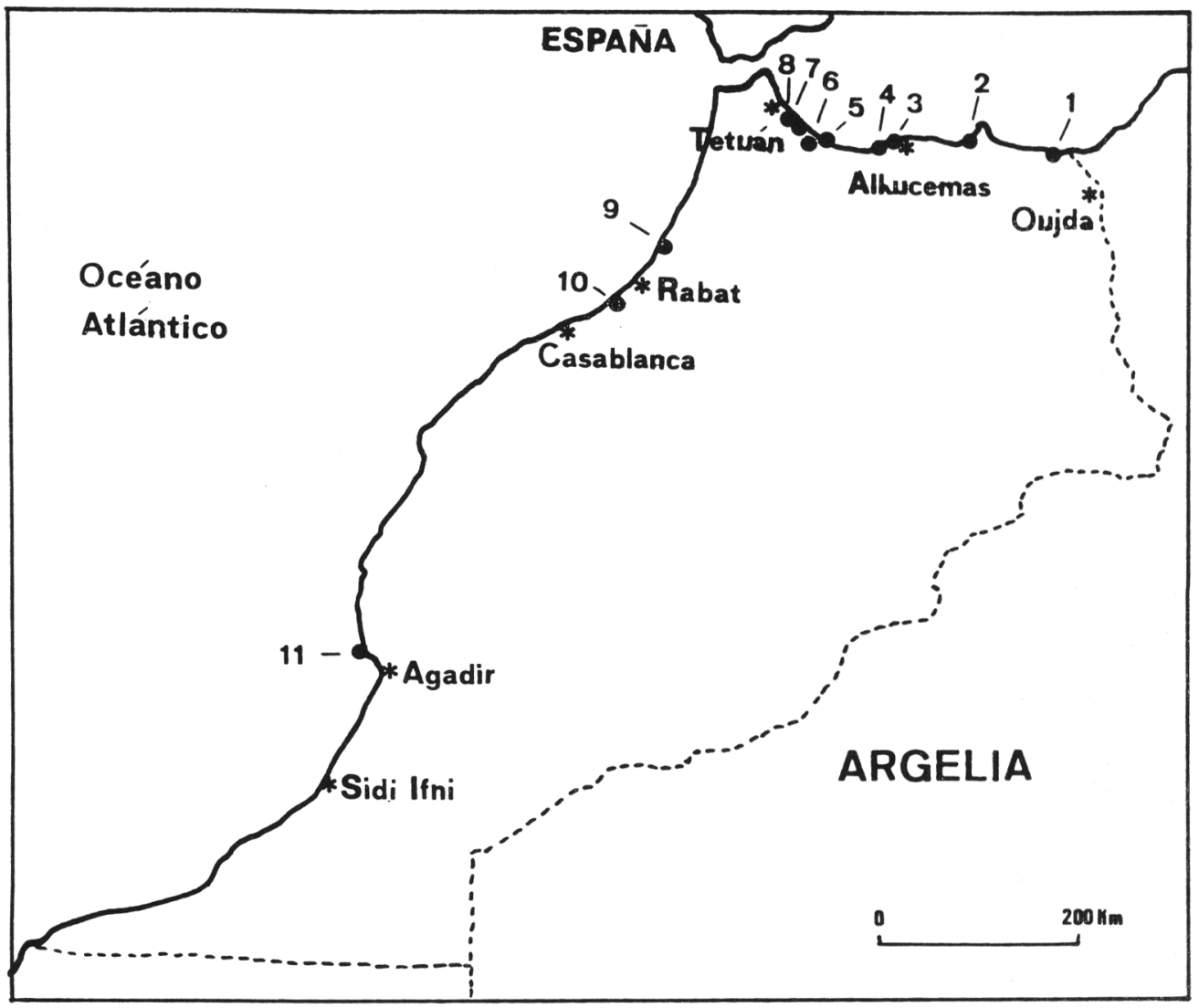

Figura 1. Localidades estudiadas. Localities studied: 1 Saidia. 2 Nador, Cabo de Charrana. 3 Al-Hoceima, Club Mediterráneo. 4 Al-Hoceima, Torres de Alcalá. 5 Dar M’Ter. 6 Tetuán, Cap Mazari. 7 Tetuán, Cap Negro. 8 Tetuán, Residencial Al-Andalus. 9 Kenitra. 10 Rabat, Bouznika. 11 Agadir, Cap Rhir.

\section{MATERIAL Y MÉTODO}

El estudio se basa en el material recolectado por los autores en diversas campañas realizadas, entre 1985-1990, en Marruecos. Los ejemplares han quedado depositados en el herbario de la Universidad de Murcia (MUB).

Para la identificación del material se han utilizado las claves de uso general (Poelt, 1969; Clauzade \& Roux 1985; Purvis et al. 1992). La nomenclatura está de acuerdo con Nimis (1993).

En el catálogo florístico se disponen los taxones por orden alfabético de géneros y especies. En el caso de los taxones poco conocidos en Marruecos se indican las citas previas.

\section{CATÁlOGO FLORÍSTICO}

Amandinea punctata (Hoffm.) Coppins \& Scheid. Sin.: Buellia punctata (Hoffm.) A. Massal.

Tetuán, Residencial Al-Andalus, sobre Ficus carica (MUB 22367). Bouznika, sobre Pistacia lentiscus (MUB 22368).

Arthonia cinnabarina (DC.) Wallr.

Kenitra, sobre Quercus suber (MUB 22330). 
Arthonia dispersa (Schrad.) Nyl.

Tetuán, Residencial Al-Andalus, sobre Ficus carica (MUB 22331).

Arthonia excipienda ( $\mathrm{Nyl}$.) Leighton

Sin.: A. dispersa (Schrad.) Nyl. var. excipienda (Nyl.) H. Oliv.

Bouznika, sobre Pistacia lentiscus (MUB 22332). Citado del Rif y Bouznika (Torrente y Egea, 1987).

Arthonia melanophthalma Dufour

Al-Hoceima, Torres de Alcalá, sobre Tetraclinis articulata (MUB 22335). Dar-M'Ter, sobre Pistacia lentiscus (MUB 22336).

Arthonia muscigena Th. Fr.

Sin.: A. exilis auct.

Al-Hoceima, Torres de Alcalá, sobre Tamarix $s p$ (MUB 22333). Saidia, sobre Juniperus phoenicea (MUB 22334). Citado de Tánger, Rabat, Safi (Torrente y Egea, 1987).

Arthonia punctiformis Ach.

Al-Hoceima, Torres de Alcalá, sobre Tamarix sp (MUB 22337). Kenitra, sobre Quercus suber (MUB 22338). Bouznika, sobre Pistacia lentiscus (MUB 22339). Agadir, sobre Argania spinosa (MUB 22340).

Arthopyrenia punctiformis (Pers.) A. Massal.

Saidia, sobre Pistacia lentiscus (MUB 22341).

Tetuán: Cabo Mazari, sobre Quercus coccifera (MUB 22342); Cabo Negro, sobre Pistacia lentiscus (MUB 22343). Bouznika, sobre Olea europaea (MUB 22344).

Arthothelium crozalsianum (De Lesd.) De Lesd.

Al-Hoceima: Torres de Alcalá, sobre Pistacia lentiscus (MUB 22345). Dar-M'Ter, sobre Tetraclinis articulata (MUB 22346). Tetuán: Cabo Mazari, sobre Quercus coccifera (MUB 22347); Residencial Al-Andalus, sobre Ficus carica (MUB 22348). Bouznika, sobre Olea europaea (MUB 22349). Agadir, sobre Periploca sp (MUB 22350). Citado de Rabat y Nador (Torrente y Egea, 1987).

Arthothelium sardoum Bagl.

Dar-M'Ter, sobre Olea europaea (MUB 22351). Bouznika, sobre Olea europaea (MUB 22352). Nueva cita para Marruecos.
Arthrosporum populorum A. Massal.

Sin.: Arthrosporum accline (Flot.) A. Massal.; Bacidia acclinis (Flot.) Zahlbr.

Dar-M'Ter, sobre Olea europaea (MUB 22353). Tetuán: Cabo Negro, sobre Eucaliptus sp (MUB 22354). Citado de Rabat, Safi y Nador (Torrente y Egea, 1987).

Bacidia globulosa (Flörke) Hafellner \& V. Wirth Sin.: Catillaria globulosa (Flörke) Th. Fr.

Al-Hoceima, Torres de Alcalá, sobre Tamarix sp (MUB 22386).

Bacidia igniarii (Nyl.) Oxner

Al-Hoceima, Torres de Alcalá, sobre Pistacia lentiscus (MUB 22356). Dar-M'Ter, sobre Pistacia lentiscus (MUB 22357). Nueva cita para Marruecos.

Bacidia laurocerasi (Duby) Zahlbr.

Tetuán, Cabo Negro, sobre Eucaliptus sp (MUB 22358). Nueva cita para Marruecos.

Bacidina phacodes (Körb.) Vezda

Sin.: Bacidia phacodes Körb.

Saidia, sobre Juniperus phoenicea (MUB 22359). Al-Hoceima, Torres de Alcalá, sobre Pistacia lentiscus (MUB 22360). Nueva cita para Marruecos.

Bactrospora patellarioides (Nyl.) Almq. var. patellarioides

Saidia, sobre Pistacia lentiscus (MUB 22361). Al-Hoceima, Torres de Alcalá, sobre Tetraclinis articulata (MUB 22362). Dar-M'Ter, sobre Olea europaea (MUB 22363). Agadir, sobre Argania spinosa (MUB 22364).

Bactrospora patellarioides (Nyl.) Almq. var. convexa (De Lesd.) Egea \& Torrente

Tetuán, Cabo Negro, sobre Eucaliptus sp (MUB 22365). Nueva cita para Marruecos.

Buellia alboatra (Hoffm.) Th. Fr.

Sin.: Diplotomma alboatrum (Hoffm.) Flot.

Agadir, sobre Argania spinosa (MUB 22366).

Caloplaca aegatica Giralt, Nimis \& Poelt

Sin.: C. quercina auct.

Nador, sobre Punica granatum (MUB 22369). Al-Hoceima, Torres de Alcalá, sobre Tetraclinis 
articulata (MUB 22370). Dar-M'Ter, sobre Pistacia lentiscus (MUB 22371). Tetuán, Residencial AlAndalus, sobre Ficus carica (MUB 22372).

Caloplaca cerina (Ehrh. ex Hedw.) Th. Fr. Kenitra, sobre Quercus suber (MUB 22373).

\section{Caloplaca ferruginea (Huds.) Th. Fr.}

Nador, sobre Punica granatum (MUB 22374).

Al-Hoceima, Torres de Alcalá, sobre Pistacia lentiscus (MUB 22375). Agadir, sobre Argania spinosa (MUB 22376).

Caloplaca holocarpa (Ach.) A. E. Wade

Saidia, sobre Pistacia lentiscus (MUB 22377). Nador, sobre Punica granatum (MUB 22378). AlHoceima: Club Mediterráneo, sobre Ficus carica (MUB 22379); Torres de Alcalá, sobre Tamarix sp (MUB 22380). Dar-M'Ter, sobre Pistacia lentiscus (MUB 22381). Tetuán, Cabo Mazari, sobre Ceratonia siliqua (MUB 22382). Bouznika, sobre Olea europaea (MUB 22383). Agadir, sobre Periploca sp (MUB 22384).

Caloplaca pollinii (A. Massal.) Jatta

Dar-M'Ter, sobre Pistacia lentiscus (MUB 22385). Citado de Tánger (Torrente y Egea, 1987)

\section{Catillaria nigroclavata (Nyl.) Schuler}

Tetuán, Cabo Negro, sobre Eucaliptus sp (MUB 22387). Kenitra, sobre Quercus suber (MUB 22388). Citado de Rabat (Werner, 1969) y Rif (Werner, 1979).

\section{Cladonia coniocracea (Flörke) Spreng.}

Tetuán, Cabo Negro, sobre Pinus sp (MUB 22389). Nueva cita para Marruecos.

\section{Diploicia canescens (Dickson) A. Massal.}

Saidia, sobre Pistacia lentiscus (MUB 22390). Bouznika, sobre Pinus pinea (MUB 22391). Agadir, sobre Argania spinosa (MUB 22392).

\section{Dirina ceratoniae (Ach.) Fr.}

Saidia, sobre Pistacia lentiscus (MUB 22393). Nador, sobre Punica granatum (MUB 22394). AlHoceima: Club Mediterráneo, sobre Ficus carica (MUB 22395); Torres de Alcalá, sobre Tetraclinis articulata (MUB 22396). Dar-M'Ter, sobre Pistacia lentiscus (MUB 22397). Tetuán, Cabo Mazari, sobre
Ceratonia siliqua (MUB 22398). Bouznika, sobre Pistacia lentiscus (MUB 22399). Agadir, sobre Argania spinosa (MUB 22400).

Lecania cyrtella (Ach.) Th. Fr.

Saidia, sobre Pistacia lentiscus (MUB 22402). Al-Hoceima, Torres de Alcalá, sobre Tamarix $s p$ (MUB 22403).

\section{Lecanora chlarotera Nyl.}

Al-Hoceima, Torres de Alcalá, sobre Tetraclinis articulata (MUB 22404). Dar-M'Ter, sobre Pistacia lentiscus (MUB 22405). Tetuán: Cabo Mazari, sobre Quercus coccifera (MUB 22406); Residencial AlAndalus, sobre Ficus carica (MUB 22407); Cabo Negro, sobre Quercus coccifera (MUB 22408).

\section{Lecanora hagenii (Ach.) Ach.}

Tetuán, Cabo Negro, sobre Quercus coccifera (MUB 22409).

\section{Lecanora horiza (Ach.) Lindsay}

Sin.: L. sienae De Lesd.

Nador, sobre Punica granatum (MUB 22410). Al-Hoceima, Club Mediterráneo, sobre Ficus carica (MUB 22412). Tetuán, Residencial Al-Andalus, sobre Ficus carica (MUB 22412). Kenitra, sobre Quercus suber (MUB 22413). Taxón frecuente en la Región Mediterránea, no citado previamente de Marruecos.

\section{Lecidella elaeochroma (Ach.) Hazsl.}

Al-Hoceima, Club Mediterráneo, sobre Ficus carica (MUB 22414). Dar-M'Ter, sobre Pistacia lentiscus (MUB 22415). Tetuán: Cabo Mazari, sobre Quercus coccifera (MUB 22416); Residencial AlAndalus, sobre Ficus carica (MUB 22417); Cabo Negro, sobre (MUB 22417). Kenitra, sobre Quercus suber (MUB 22419). Bouznika, sobre Olea europaea (MUB 22420).

Leptorhaphis aff. opunticola Fiol \& Aguirre

Bouznika, sobre Pistacia lentiscus (MUB 22421). Por la forma y tamaño de los ascomas y la reacción I- del hamatecio nuestro taxón es próximo a Leptorhaphis opunticola. Sin embargo, en $L$. opunticola las esporas son menores (25-32.5 x 1.6 $2.5 \mu \mathrm{m}$; Aguirre-Hudson \& Fiol 1993) que en el 
ejemplar de Marruecos $(32-38$ x 2.5-3.5 $\mu \mathrm{m})$.

Parmelia subaurifera Nyl.

Kenitra, sobre Quercus suber (MUB 22424).

Parmotrema chinense (Osbeck) Hale \& Athi

Sin.: Parmelia perlata (Hudson) Ach., P. coniocarpa Laurer

Kenitra, sobre Quercus suber (MUB 22422).

Parmotrema hypoleucinum (J. Steiner) Hale

Sin.: Parmelia hypoleucina J. Steiner, P. subincana Mah. et Gill.

Tetuán, Cabo Negro, sobre Pinus halepensis (MUB 22423).

Pertusaria coccodes (Ach.) Nyl.

Tetuán, Cabo Negro, sobre Eucaliptus sp (MUB 22425). Nueva cita para Marruecos.

Pertusaria heterochroa (Müll. Arg.) Erichsen

Dar-M'Ter, sobre Pistacia lentiscus (MUB 22426). Tetuán: Cabo Mazari, sobre Ceratonia siliqua (MUB 22427); Residencial Al-Andalus, sobre Ficus carica (MUB 22428); Cabo Negro, sobre Quercus coccifera (MUB 22429).

Pertusaria leioplaca DC.

Sin.: P. leucostoma A. Massal.

Tetuán, Cabo Negro, sobre Quercus coccifera (MUB 22430). Kenitra, sobre Quercus suber (MUB 22431).

Pertusaria pertusa auct.

Dar-M'Ter, sobre Tetraclinis articulata (MUB 22432).

Physcia adscendens (Fr.) H. Olivier

Dar-M'Ter, sobre Tetraclinis articulata (MUB 22433). Kenitra, sobre Quercus suber (MUB 22434).

Physconia enteroxantha (Nyl.) Poelt

Tetuán, Cabo Negro, sobre Pinus sp (MUB 22435). Nueva cita para Marruecos.

Porina aenea (Wallr.) Zahlbr.

Sin.: P. carpinea (Pers. ex Ach.) Zahlbr.

Tetuán, Cabo Mazari, sobre Quercus coccifera (MUB 22436); Cabo Negro, sobre Pistacia lentiscus (MUB 22437). Citado del Rif (Torrente \& Egea 1987).
Ramalina canariensis J. Steiner

Dar-M'Ter, sobre Olea europaea (MUB 22438). Tetuán: Cabo Negro, sobre Pinus halepensis (MUB 22439). Kenitra, sobre Quercus suber (MUB 22440).

Ramalina farinacea (L.) Ach.

Kenitra, sobre Quercus suber (MUB 22444).

Ramalina fraxinea (L.) Ach.

Agadir, sobre Argania spinosa (MUB 22445).

Ramalina maciformis (Delile) Bory

Sin.: R. evernioides Nyl.

Saidia, sobre Pistacia lentiscus (MUB 22441). Al-Hoceima, Torres de Alcalá, sobre Pistacia lentiscus (MUB 22442). Agadir, sobre Argania spinosa (MUB 22443).

Ramalina pusilla Le Prévost. ex Duby

Tetuán, Cabo Negro, sobre Pinus halepensis (MUB 22446).

Rinodina anomala (Zahlbr.) H. Mayrhofer \& Giralt Sin.: Buellia anomala Zahlbr.

Saidia, sobre Juniperus phoenicea (MUB 22486). Tetuán: Cabo Mazari, sobre Quercus coccifera (MUB 22447); Residencial Al-Andalus, sobre Ficus carica (MUB 22487); Cabo Negro, sobre Pinus halepensis (MUB 22448). Nueva cita para Marruecos.

Rinodina gennarii Bagl.

Tetuán, Cabo Negro, sobre Pinus halepensis (MUB 22449).

Rinodina oleae Bagl.

Saidia, sobre Juniperus phoenicea (MUB 22450). Nueva cita para Marruecos.

Rinodina pruinella Bagl.

Saidia, sobre Pistacia lentiscus (MUB 22451). Al-Hoceima, Torres de Alcalá, sobre Pistacia lentiscus (MUB 22452). Bouznika, sobre Pinus pinea (MUB 22453).

Roccella phycopsis (Ach.) Ach.

Al-Hoceima, Torres de Alcalá, sobre Pistacia lentiscus (MUB 22454). Dar-M'Ter, sobre Tetraclinis articulata (MUB 22455). Agadir, sobre Argania spinosa (MUB 22456). 
Schismatomma dirinellum (Nyl.) Zahlbr.

Sin.: S. picconianum (Bagl.) J. Steiner

Al-Hoceima, Torres de Alcalá, sobre Tamarix sp (MUB 22457). Dar-M'Ter, sobre Pistacia lentiscus (MUB 22458). Tetuán: Cabo Mazari, sobre Ceratonia siliqua (MUB 22459); Residencial AlAndalus, sobre Ficus carica (MUB 22460).

Schismatomma albocinctum (Nyl.) Zahlbr. Sin.: S. pitardi (De Lesd.) Egea \& Torrrente

Al-Hoceima, Torres de Alcalá, sobre Pistacia lentiscus (MUB 22461). Dar-M'Ter, sobre Pistacia lentiscus (MUB 22462). Tetuán: Cabo Mazari, sobre Ceratonia siliqua (MUB 22463); Residencial AlAndalus, sobre Ficus carica (MUB 22464).

Schismatomma graphidioides (Leighton) Zahlbr. Sin.: S. ricasolii (A. Massal.) Egea \& Torrente Tetuán, Cabo Mazari, sobre Ceratonia siliqua (MUB 22465).

Teloschistes crysophthalmus (L.) Th. Fr. Kenitra, sobre Quercus suber (MUB 22466).

Teloschistes villosus (Ach.) Norman

Saidia, sobre Pistacia lentiscus (MUB 22467). Bouznika, sobre Pinus pinea (MUB 22468). Agadir, sobre Argania spinosa (MUB 22469).

\section{Thelenella modesta (Nyl.) Nyl.}

Sin.: Microglaena modesta (Nyl.) A. L. Smith.

Saidia, sobre Pistacia lentiscus (MUB 22470).

Dar-M'Ter, sobre Pistacia lentiscus (MUB 22471). Tetuán, Cabo Mazari, sobre Quercus coccifera (MUB 22472).

\section{Thelopsis isiaca Stizenb.}

Saidia, sobre Pistacia lentiscus (MUB 22473). Nador, sobre Punica granatum (MUB 22474). AlHoceima: Club Mediterráneo, sobre Ficus carica (MUB 22475); Torres de Alcalá, sobre Pistacia lentiscus (MUB 22476). Agadir, sobre Lycium sp (MUB 22477).

Xanthoria parietina (L.) Th. Fr.

Saidia, sobre Pistacia lentiscus (MUB 22478). Nador, sobre Punica granatum (MUB 22479). AlHoceima: Club Mediterráneo, sobre Ficus carica (MUB 22480); Torres de Alcalá, sobre Pistacia lentiscus (MUB 22481). Dar-M'Ter, sobre Pistacia lentiscus (MUB 22482). Tetuán: Cabo Mazari, sobre Quercus coccifera (MUB 22483); Residencial AlAndalus, sobre Ficus carica (MUB 22483). Bouznika, sobre Pinus pinea (MUB 22485).

AGRADECIMIENTOS. Agradecemos a la Dra Aguirre-Hudson la revisión de Leptorhaphis, al Dr Coppins la de algunos ejemplares de Bacidia y a la Dra Giralt la de algunos ejemplares de Arthothelium y Rinodina.

\section{BIBLIOGRAFÍA}

AGUIRRE-HUDSON, B.\&L.FIOL-1993-A new species of Leptorhaphis (Arthopyreniaceae) on Opuntia from the Balearic Islands. Lichenologist 25: 207-210.

ALONSO, F.L. y J.M. EGEA -1994- Líquenes calcícolas y terrícolas de algunas localidades costeras de Marruecos. Acta Bot. Malacitana 19: 51-61.

CLAUZADE, G. \& C.ROUX-1985-Likenoj de Okcidenta Europo Ilustrita determinlibro. Bull. Soc. bot. CentreOuest, $n^{o}$ spéc. 7: 1-893.

EGEA, J.M. -1996- Catalogue of lichenized and lichenicolous fungi of Marocco. Bocconea 6: 19-114.

NIMIS, P.L. - 1993- The lichens of Italy. Museo Regionale de Science Naturali. Torino.

POELT, J. -1969- Bestimmungsschlüssel europäischer Flechten. J. Cramer. Vaduz.

PURVIS, O.W., B.J. COPPINS, D.L. HAWKSWORTH, P.W. JAMES \& D.M. MOORE (eds.) -1992- The Lichen Flora of Great Britain and Ireland. Nat. Hist. Mus. Publ. London.

TORRENTE, P. y J.M. EGEA -1987- Prospecciones liquenológicas en Africa del Norte II. Líquenes calcícolas y epífitos del litoral de Marruecos. Anales Biología 13 (Biología Vegetal 3): 15-19.

WERNER, R.G. -1969- Lichenes et fungi maroccani III. Etude Substratique. Bull. Soc. Mycol. France 85 (2): 195-204.

WERNER, R.G. - 1979- La flore lichénique de la cordillère bético-rifaine.Étude phytogéographiqueetécologique. Collect. Bot. 11: 401-463.

Aceptado para su publicación en Octubre de 1997

Dirección de los autores. Departamento de Biología Vegetal (Botánica), Facultad de Biología, Universidad de Murcia, Campus de Espinardo. 30071, Murcia, Spain. 